\title{
PERCUTANEOUS INTERVENTION TREATMENT OF GIANT EXTRARENAL ANEURYSM ARTERIOVENOUS FISTULA
}

\author{
Nguyen The Huy ${ }^{1}$, Phan Thao Nguyen ${ }^{1}$, Tran Thuy Nguyen ${ }^{1,2 *}$, Le Ngoc Thanh ${ }^{1,2}$
}

\begin{abstract}
Objective: Anteriovenous fistula is a highflow vascular malformation. Anteriovenous fistula is characterized by a direct connection between an artery and a vein without the presence of a nidus (network of arterial and venous channels). Renal artery aneurysm concomitant with a renal arteriovenous fistula is extremely rare.

Case report: We reported the case of a 45year-old female who has giant renal artery aneurysm combined with high-flow RAVF who presented with gross hematuria. The patient's embolization of a giant extrarenal aneurism arteriovenous fistula was successfully treated using specialized coils and plug device.

Conclusions: Renal arteriovenous fistula is a disease that can lead to serious complications. Treatment of this condition should be initiated promptly after diagnosis. Embolization endovascular is a feasible, efficient and safe method which can maximally retain normal renal function.
\end{abstract}

Key words: renal arteriovenous fistula, renal artery aneurysm, hematuria.

\section{INTRODUCTION}

Renal arteriovenous fistula is a rare lesion, its prevalence has been reporter to be less than $0.04 \%$. Furthermore, renal artery aneurysm concomitant with a renal arteriovenous fistula is extremely rare. It has a considerable clinical impact on hypertension, hematuria, abdominal pain and high-output cardiac failure. Three main subtypes are generally classified based on etiology: acquired, idiopathic or arising in a congenital arteriovenous malformation. In recent years, percutaneous intervention embolization has become the first choice for the treatment of this disorder. The biggest advantage of endovascular treatment is a less invasive alternative to traditional open surgery $[1,5]$. is extremely rare. This study highlights a case of renal artery aneurysm concomitant with a renal arteriovenous fistula which is extremely rare, and discusses further the diagnosis, treatment and other clinical considerations.

\section{CASE REPORT}

A 45 years-old female was admitted to our hospital because of gross hematuria. In history, she had hypertension for the duration of 5 years (up to $180 / 100 \mathrm{mmHg}$ - poor control of blood pressure). She was found to have a left renal AVF by color doppler ultrasonography seven years ago. Sometimes, she has pain lower back and saw a litte bood in urine. Past medical history was unremarkable, but there was a family history of hypertention. No history of abdominal trauma, renal biopsy, or intervention to the renal vascular system.

1 Cardiovascular Center, E hospital, Viet Nam

2 University of Medicine and Pharmacy, Vietnam National University, Viet Nam

*Corresponding author: Nguyen Tran Thuy

Email: drtranthuyvd@gmail.com

Address: E Hospital, 87-89 Tran Cung, Hanoi, Vietnam, 10000

Vietnam National University, 114 Xuan Thuy, Hanoi, Vietnam, 10000

Received: 24 June 2021

Accepted: 05 July 2021 
In the morning, she felt severe pain in the left lumbar region. She presented to our hospital with massive hematuria and passage of blood clots in urine. Her blood pressure at the time of presentation was $105 / 60 \mathrm{mmHg}$, heart rate was 100 beat /minute. She was pallor, soft abdomen, palpaple mass in the left lumbar region. Blood analysis showed hemoglobin $7.4 \mathrm{~g} / \mathrm{dl}$ (normal range: 12.0 to $16.0 \mathrm{~g} / \mathrm{dl}$ ), hematocrit $22.4 \%$ (normal range: 36 to $46 \%$ ), red blood cells 2.7 $\mathrm{T} / 1$ (normal range: 4.0 to $5.2 \mathrm{~T} / 1$ ). Her biochemical and coagulation parameter were within normal limits. Urine analysis showed plenty of red blood cells. Ultrasound of the abdomen and CT scanner abdomen was advised.

Abdominal ultrasonography demonstrated the size of right and left kidney being 101 and $98 \mathrm{~mm}$, respectively. Color doppler sonography showed a vascular malformation in the left inferior renal pole. A giant extrarenal aneurism measuring 55*65 mm. The spectral Doppler tracings showed a high-velocity, low resistance flow within the artery and turbulent, pulsatile, arterialized flow in the segmental dranining vein [figure 1].

An abdominal three-dimensional CT scan shown in Figure 2. A huge, ball-shaped renal artery anerysm had developed outside the anterior bottom of left kidney. The maxium diameter of an abnormal vascular mass was $65 \mathrm{~mm}$. The interlobar renal artery was dilated and tortous ( $10 \mathrm{~mm}$ ) [Figure 2] and the draining vein was enlarged approximately $19 \mathrm{~mm}$ [Figure 2B].

Other diagonostic imaging tests include: Chest X-ray showed Cardiomegaly with a Cardiothoracic ratio of 55\%, no pleural effusion. Electrocardiograms showed sinus rhythm, no left ventricular hypertrophy (R V5+S V1 amp $=3.0 \mathrm{mV})$. Transthoracic 2-dimensional echocardiography demonstrated mild mitral regurgitation. The left ventricle was dilated, with an end-diastolic diameter (LVDd) of $58 \mathrm{~mm}$. The left ventricular mass was $192 \mathrm{~g}$ (calculated following the equation by Devereux) - BSA: 1.47 $\mathrm{m}^{2}$. The left ventricular mass index (normalised for body surface area) was $130 \mathrm{~g} / \mathrm{m}^{2}$. The wall motion was normal. Echocardiography revealed a left ventricular ejection fraction of $68 \%$. The pulmonary artery pressure is $15 \mathrm{mmHg}$.

The patient was transfused with two additional blood units (blood type $\mathrm{O}^{+}$). Her hemodynamic status became stable. The patient has giant renal artery aneurysm combined with high-flow renal arteriovenous fistula and massive hematuria. After consultation with team of interventional doctors and surgeons, we decided to manage the arteriovenous fistula with embolization.

Procedural step: A 6F diagnostic JR catheter (Terumo Inc, Tokyo, Japan) was placed by Seldinger technique though the right brachial artery under local anesthesia. Angiogram selective left renal artery showed enlarged left interlobar renal artery branch with increased blood flow velocity and volume. This artery drained directly into a giant renal aneurysm and pouring into the drainage vein, the left renal vein, and the inferior vena cava [figure 3]. The Left renal artery and vein displayed almost simultaneously and the feeding artery of the fistula was uniformly dilated and tortuous. ALeft renal artery was engaged with Fortress 6F $100 \mathrm{~cm}$ guide catheter (Biotronik Inc, Germany) access though the right brachial artery. We tried push deeply catheter in interlobar renal artery. The left interlobar renal artery was very tortous so it is very hardly to push the catheter. We used both $\mathrm{J}-$ Tip 0.035 inch (Terumo Inc, Tokyo, Japan) and 
stiff J - Tip 0.035 inch (Terumo inc, Tokyo, Japan) to support catheter. Finally, Catheter Fortress was deeply approached close to aneuryms. A $20 \mathrm{~mm}$ Amplatzer vascular plug II (Abbott Ins, USA) was taken; a device with the delivery cable was introduced through the delivery sheath and negotiated up to the mouth of a giant aneurysm. We confirmed proper positioning and landing zone base a non-selective renal contrast injection though JR catheter $6 \mathrm{~F}$. The vascular plug II was deployed in the left segmental renal artery. Post-deployment contrast injection showed mild flow in an aneurysm. We decided to use coils. A separate microcatheter was placed at the front of vascular plug and two Ruby Coil (16 $\mathrm{mm} * 60 \mathrm{~cm}$ and $20 \mathrm{~mm} * 60 \mathrm{~cm}$ ) (Penumbra Inc, California, USA). After 10 minutes, A selective left renal contrast injection showed totally occlusion left interlobar renal artery, no flow in an aneurysm [figure 4]. Completion angiography demonstrates a near-complete nephrogram with excellent peripheral flow.

Post procedure, the patient was monitored in the postoperative room for two days. She had no chills, fever or other symtoms postoperatively. Hematuria completely stopped after two days. No obvious abnormalities were found on urinalysis and renal funtion test (creatinin $56 \mu \mathrm{mol} / \mathrm{L}$; range $45-84 \mu \mathrm{mol} / \mathrm{L}$ ). Color doppler ultrasonography showed that no blood flow signals were found in the vasculature of the AVF. The patient's symptoms of lumbago gradually subsided after two weeks.

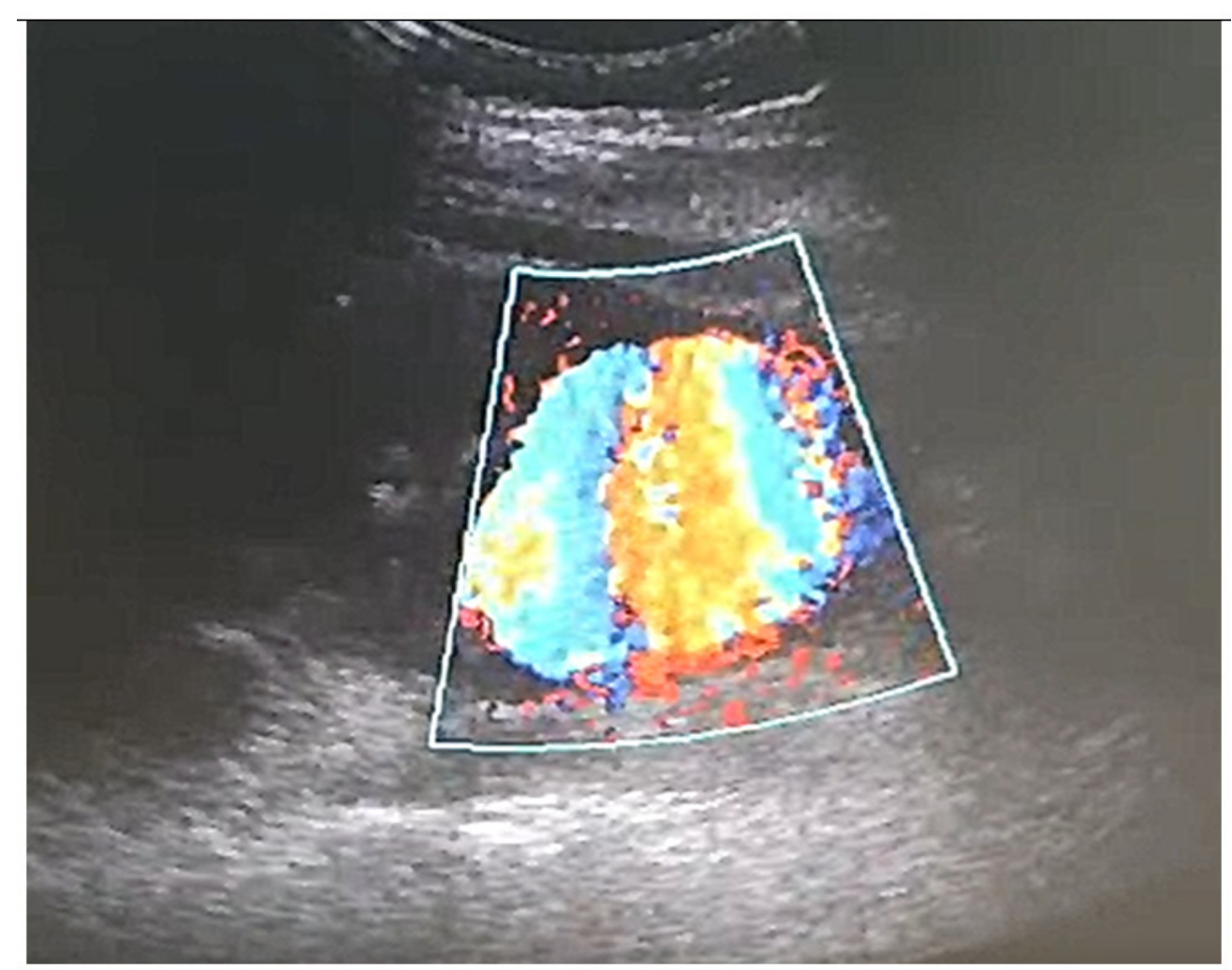

Figure 1: Color Doppler showed a high-velocity, turbulent in aneuryms 


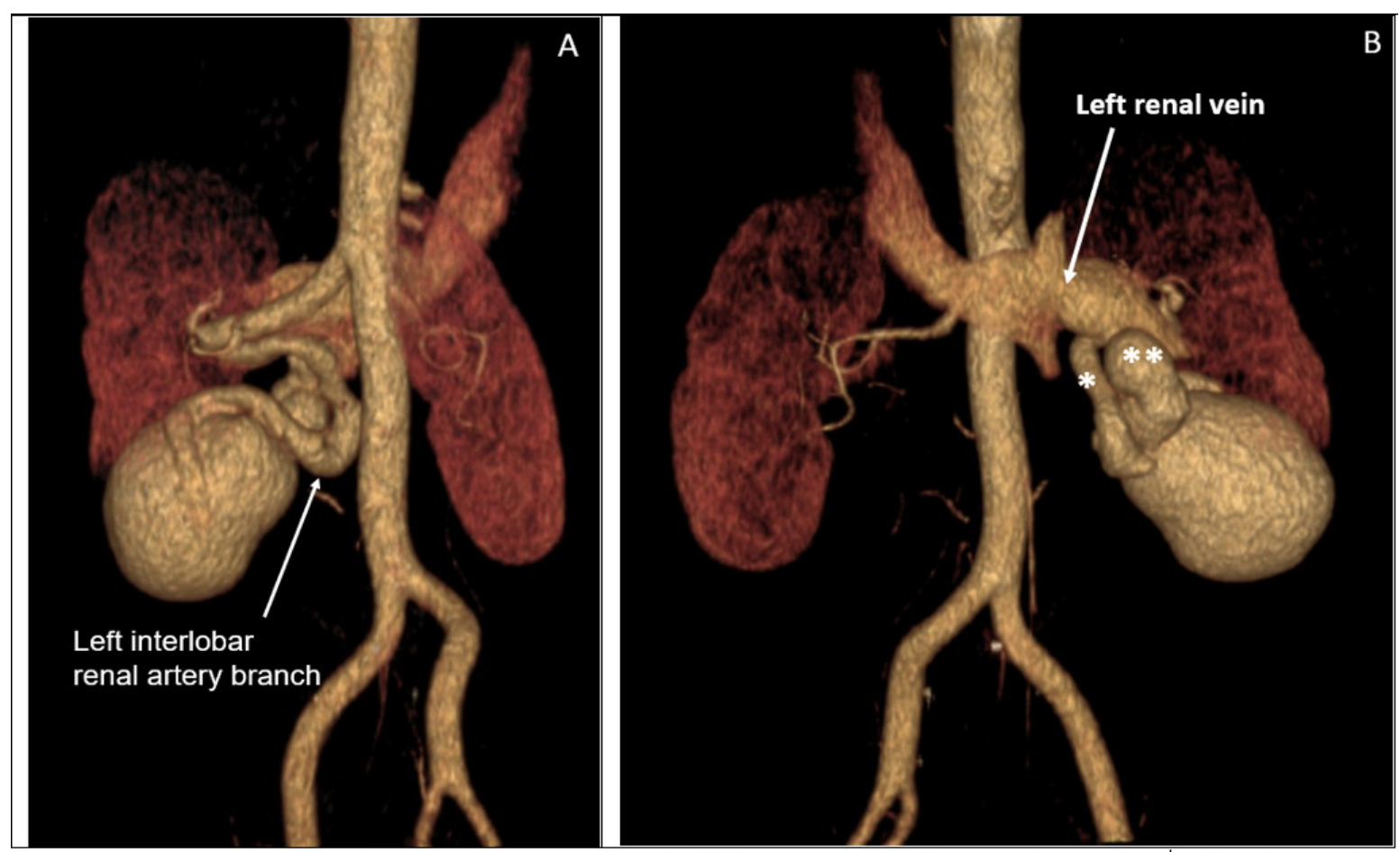

Figure 2: Abdominal three-dimensinal CT scan. A: Posterior view. B: Anterior view * left interlobar renal artery branch, ** left interlobar renal vein.

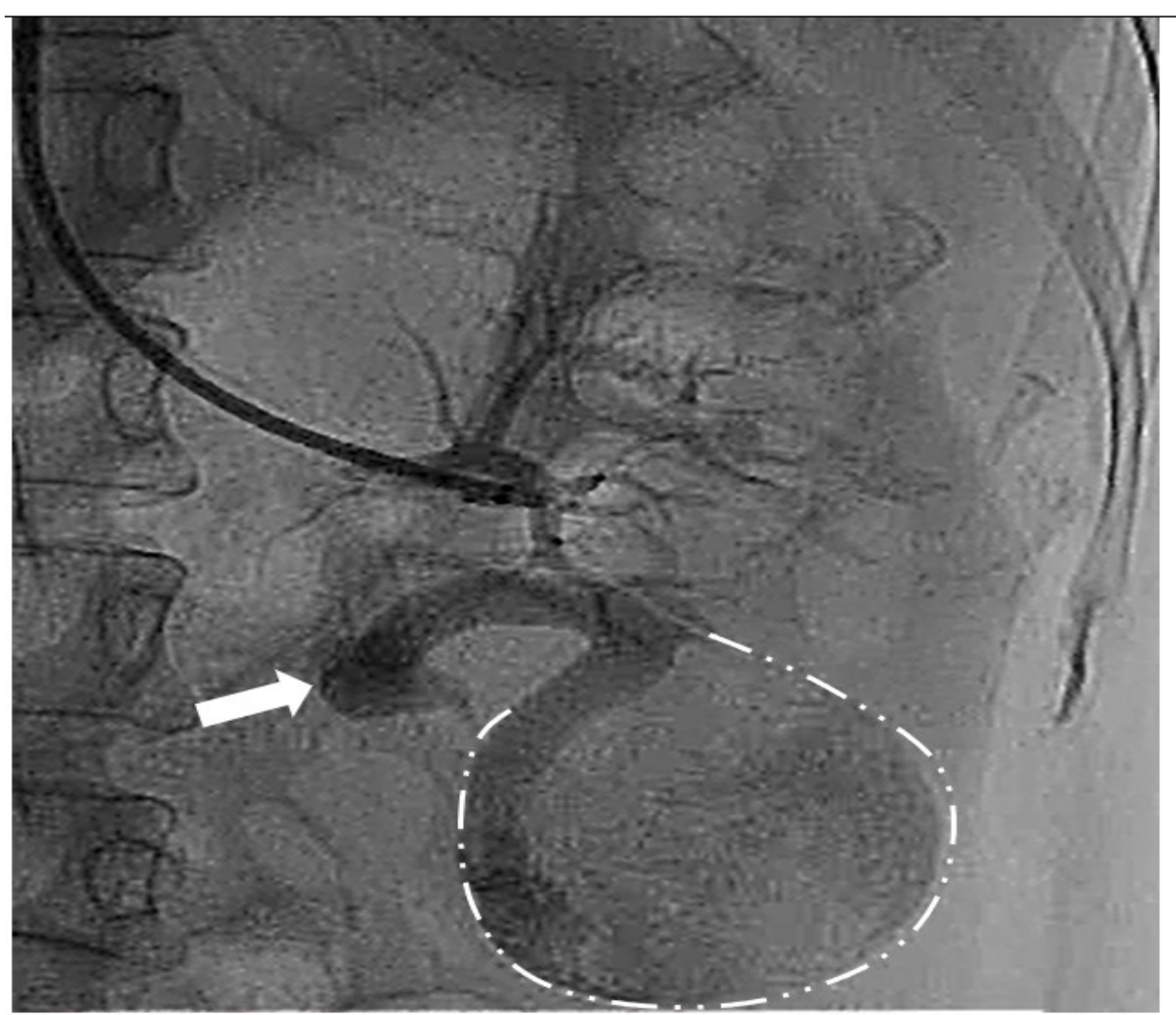

Figure 3: Selective angiogram left renal artery. Arrow: left interlobar renal artery branch. Dashed line: renal artery aneurysm.| 


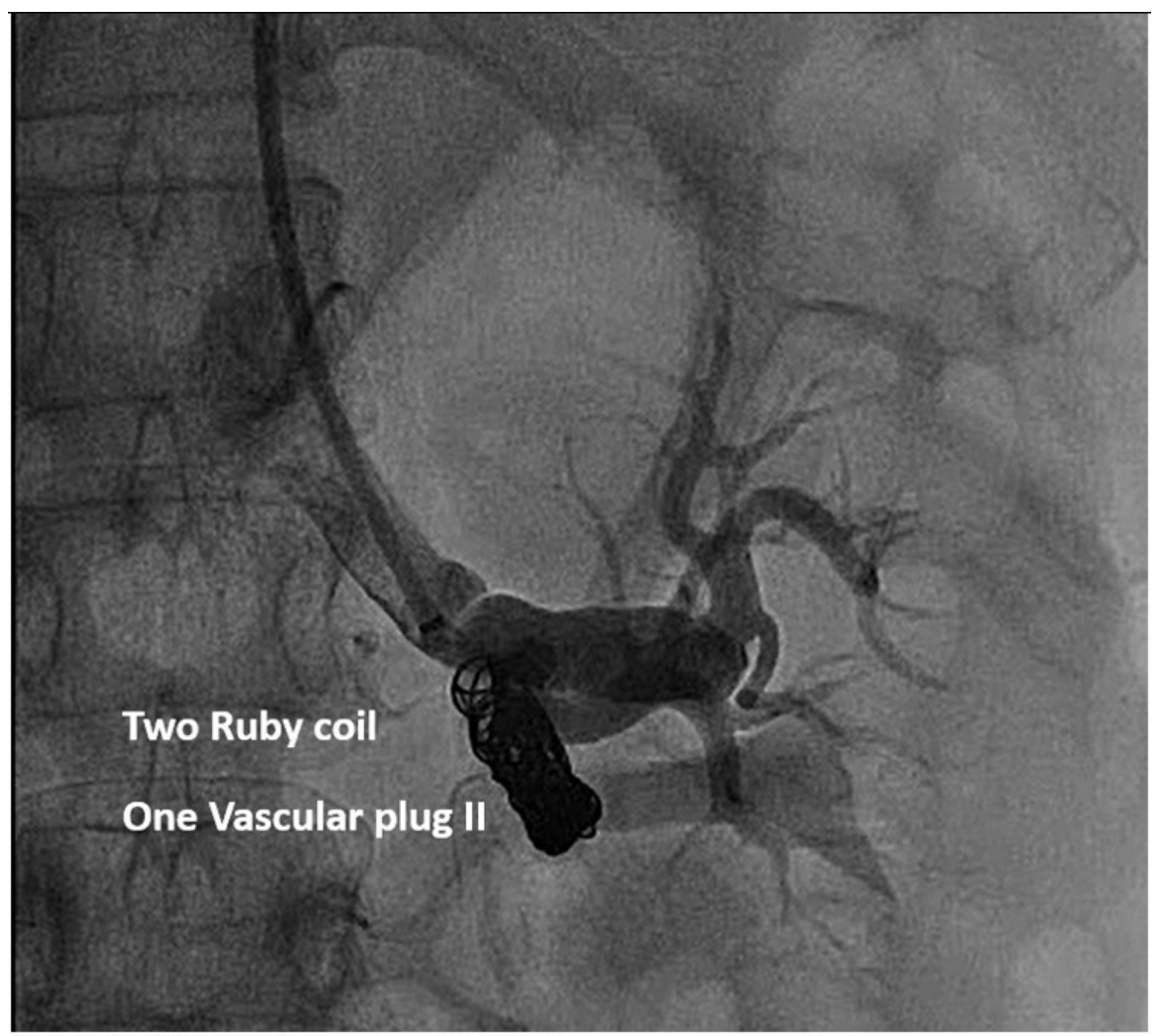

Figure 4: Selective Angiogram left renal artery post procedure.

\section{DISCUSSION}

Anteriovenous fistula is a high-flow vascular malformation. Anteriovenous fistulas are characterized by a direct connection between an artery and a vein without the presence of a nidus (network of arterial and venous channels) (Rueth). The causes of renal AVFs are normally classified as congenital (14\% - 27\%), idiophathic (3\%) and acquired characteristics (70\% - 80\%). Some frequent cause in acquired AVFs include: needle biopsy, injury, surgery, inflammation, infection, and due to the presence of malignant tumors. In addition, with the more widespread use of interventional procedures such as percutaneous needle biopsy and percutaneous nephrostomy, iatrogenic fistulas have become more frequent. In our patient there was no clinical evidence indicating the development of iatrogenic or traumatic arteriovenous fistula, the lesion was classified as congenital or idiopathic [5].
Clinical manifestations of the arteriovenous fistulas depend on their localisation and size. The most frequent clinical signs are: haematuria, mild tachycardia, Hypertension and symptoms of congestive heart failure. It has been reported that a bruit is less frequent in cirsoid arteriovenous malformations and haematuria is more common in congenital malformations. In patients with arteriovenous fistula, increased cardiac output and hypertension may lead to the development of cardiac failure in about $40 \%$ of patients with the severity of symptoms and haemodynamic changes depending on blood flow through the fistula. In our patient congestive heart failure was not present however left ventricular was dilated and left ventricular muscle mass index was increase. It was sign of over volume loading, the possibility of future congestive heart failure was taken into consideration[1].

Gross hematuria as the primary complaint was reported in $72 \%$ of cases RAVF. Hematuria 
results from minute rupture of the thin-walled veins into the collecting system from increase in pressure. This is why even small, peripherally located AVMs can cause massive hematuria[2].

Report of many authors indicated the high effectiveness of color Doppler sonography in diagnosing and following patients with renal arteriovenous malformations. However, In order to have an appropriate treatment strategy multislice CT or Magnetic Resonance imaging is essential. Although the definite diagnosis of arteriovenous malformations must be confirmed by angiography[4].

At present, the primary treatment includes surgical approaches and interventional embolization. However, surgical approaches, such as nephrectomy or ligation of the renal artery, are traumatic and cannot achieve the aim of retaining functional nephrons. In recent years, interventional embolization of AVFs with elastic coils, gelatin sponge particles, and polyvinyl alcohol particles has become a feasible, efficient, and safe method to occlude the fistula successfully while maximally retaining renal function. However, for large high-output renal AVFs, there is a risk of emboli shifting to unexpected areas; these are therefore recommended for surgical treatment. The choice of embolization material depends on the anatomical characteristics of the blood vessel and the experience of the interventional physician. The risk of embolotherapy include: reflux of obliterating agents, loss of normal renal parenchyma, pulmonary embolism. Report of Mori et al showed that a post-embolization syndrome may occur, characterized by pain in the embolized area, nausea, vomiting and fever lasting up to 5 days [3].

\section{CONCLUSION}

Renal arteriovenous fistula is a disease that can lead to harmful complications. Treatment of this pathology should be instituted when the disease is discovered. Embolization endovascular is a feasible, efficient and safe method which can maximally retain normal renal function.

\section{FOOTNOTES}

Acknowledgements: The authors would like to thank Dr Chau Nguyen for his help with the 3D CT angiography images.

Competing interests: None declared.

Patient consent: Obtained

\section{REFERENCES}

1. Chen, X., et al., Embolization of high-output idiopathic renal arteriovenous fistula primarily using an atrial septal defect occluder via venous access: a case report. BMC nephrology, 2019. 20(1): p. 15-15.

2. Fogazzi, G.B., M. Moriggi, and U. Fontanella, Spontaneous renal arteriovenous fistula as a cause of haematuria. Nephrol Dial Transplant, 1997. 12(2): p. 350-6.

3. Mori, T., et al., Renal arteriovenous fistula with rapid blood flow successfully treated by transcatheter arterial embolization: application of interlocking detachable coil as coil anchor. Cardiovasc Intervent Radiol, 2004. 27(4): p. 374-6.

4. Nassiri, N., et al., Transarterial treatment of congenital renal arteriovenous fistulas. J Vasc Surg, 2013. 58(5): p. 1310-5.

5. Zhou, F., et al., Hypertension Caused by Renal Arteriovenous Fistula with Multiple Renal Artery Aneurysms. Ann Vasc Surg, 2021. 70: p. 565.e11-565.e13. 